\title{
Subacute Cutaneous Lupus Erythematosus: A Paraneoplastic Dermatosis in Breast Carcinoma?
}

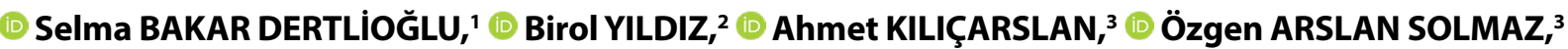 \\ (1) Pervin KARABULUT ${ }^{3}$
}

\author{
'Department of Dermatology, Elazığ Training and Research Hospital, Elazığ-Turkey \\ 2Department of Oncology, Elazığ Medical Park Hospital, Elazığ-Turkey \\ ${ }^{3}$ Department of Pathology, Elazığ Training and Research Hospital, Elazığ-Turkey
}

\begin{abstract}
SUMMARY
A 52-year-old woman presented to our clinic for the evaluation of a skin rash with a 15-month history that initially started on her face followed by the involvement of the scalp, neck, and forearm. On physical examination, nonscaly erythematous maculopapular and plaques, annular and photo-distributed eruptions involving the face, scalp, forearm, and dorsal of the hands were found. Non-scarring hair loss on the scalp and a decrease in eyebrows and eyelashes were observed. The biopsy results in combination with the clinical presentation, physical exam findings, and serologic markers established the diagnosis of subacute cutaneous lupus erythematosus associated with breast carcinoma. Low-potency topical steroid therapy was initiated for twice daily. During the patient's control examination in her third week, the Subacute cutaneous lupus erythematosus lesion was partially regressed. The patient's treatment continues in the oncology department.
\end{abstract}

Keywords: Carcinoma; paraneoplastic; subacute cutaneous lupus erythematosus.

Copyright $\odot$ 2021, Turkish Society for Radiation Oncology

\section{Introduction}

Subacute cutaneous lupus erythematosus (SCLE) is a distinct subset of systemic lupus erythematosus clinically characterized by typical psoriasiform and/or annular lesions with a mild or absent systemic involvement. Lesions are usually photosensitive, occurring in predominantly sun-exposed areas. The diagnosis is established by clinical examination, selective laboratory screening, histopathological examination, and immunoserologic findings. Photoprotection with clothing and chemical and physical sunscreens that are active on UVA and UVB radiations is very important. Topical therapy is based on the use of steroids and calcineurin inhibitors, while the systemic therapy involves the use of hydroxychloroquine as the first drug of choice.

\section{Case Report}

A 52-year-old woman presented to our clinic for the evaluation of a skin rash with a 15-month history that initially started on her face followed by the involvement of the scalp, neck and forearm. Sixteen months ago, she was diagnosed with an invasive grade II (7/9) (nuclear atypia2, tubular formation 3, mitosis 2) ductal breast carcinoma, and was treated with the Adriamisin-Siklofosfamid Neoadjuvan Protocol in sessions.

On physical examination, nonscaly erythematous maculopapular and plaques, annular and photo-distributed eruptions involving the face, scalp, forearm, and dorsal of the hands were found. Non-scarring hair loss on the scalp and a decrease in the eyebrows and eyelashes were seen (Fig. $1 \mathrm{a}-\mathrm{c}$ ). The rest of the physical examination was unremarkable.
Received: October 07, 2020

Accepted: November 19, 2020

Online: November 27, 2020

Accessible online at:

www.onkder.org

OPEN ACCESS This work is licensed under a Creative Commons

Attribution-NonCommercial 4.0 International License.
Dr. Selma BAKAR DERTLIOGĞLU

Elazığ Eğitim ve Araştırma Hastanesi,

Dermatoloji Aanabilim Dalı,

Elazığ-Turkey

E-mail: selmadertlioglu@hotmail.com 


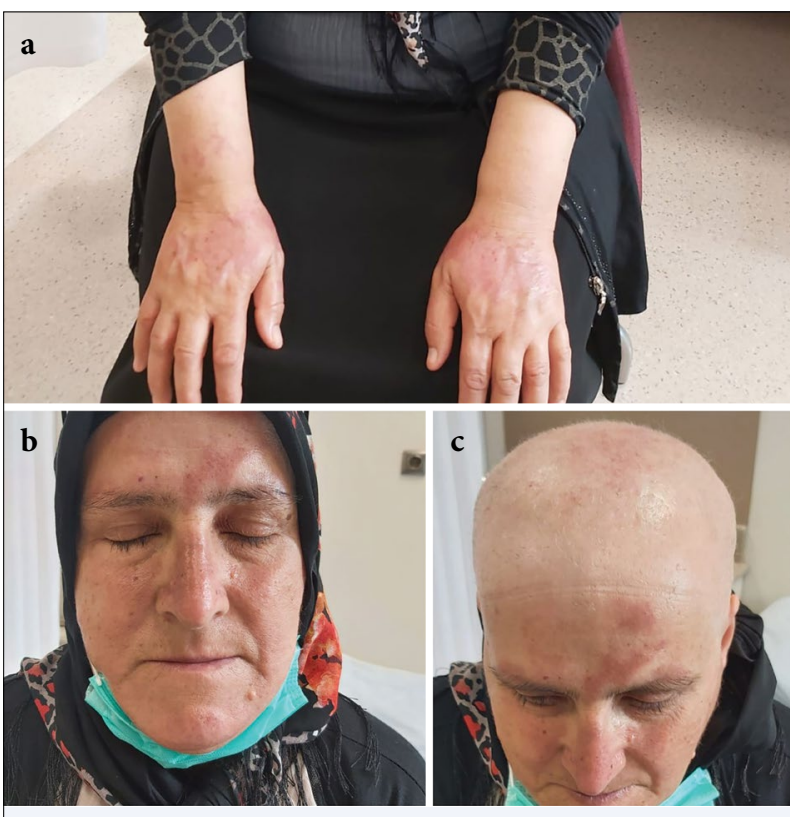

Fig. 1. (a-c Nonscaly erythematous plaques and photodistributed eruptions involving the face, forearm, and dorsal of the hands; erythematous plaques on the forehead, nose, and right cheek; and erythematous plaques on the right eyebrow, forehead, and vertex.

Histological examination of a skin biopsy found epidermal atrophy, vacuolar degeneration in the basal layer, basement-membrane thickening, superficial perivascular lymphocytic infiltration, and a mild chronic inflammatory infiltrate periadnexally and along the dermoepidermal junction, consistent with subacute cutaneous lupus erythematosus (SCLE) (Figs. $2 \mathrm{a}, \mathrm{b}, 3)$. Direct immunofluorescence yielded negative results. Serological tests were positive for extractable nuclear antigen (anti-Ro (SS-A) and anti-La (SS-B) and antinuclear antibody) and negative for rheumatoid factor.

Full blood count, urea, and electrolytes were within normal limits. Liver transaminases, bilirubin, and alkaline phosphatase were normal. There was no blood or protein in the urine. CT of the abdomen and pelvis were normal. Lymphadenopathies showing increased metabolism in the left axilla were evaluated by the PET method.

The biopsy results in combination with the clinical presentation, physical exam findings, and serologic markers established the diagnosis of SCLE associated with breast carcinoma.

It was initially thought that the patient's SCLE may have been drug induced, but this is unlikely in light of her breast cancer diagnosis and the lack of a tempo-
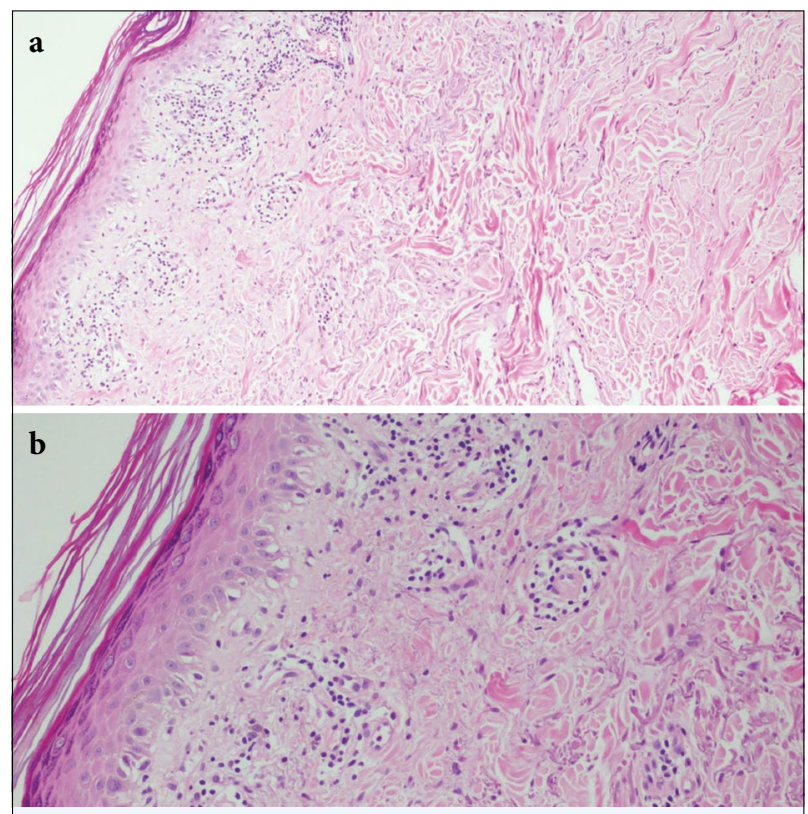

Fig. 2. (a, b) Baskety hyperkeratosis on the surface, vacuolar degeneration in the basal layer, superficial perivascular lymphocytic infiltration HEX40, HEX200.

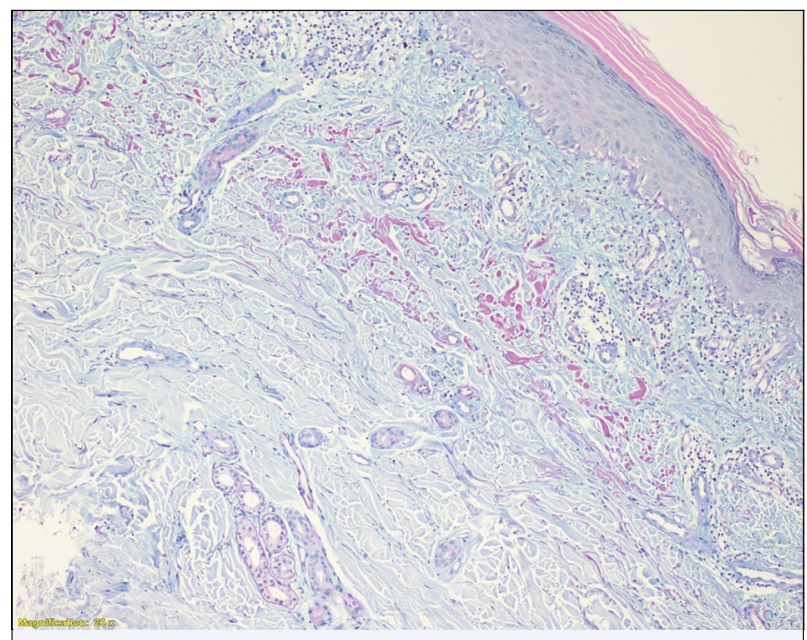

Fig. 3. PAS positive mucin deposits on the dermis.

ral relationship between the onset of the rash and her therapeutic drug regimen. Her skin lesions had started approximately one month before the initation of the chemotherapy treatment. Hence, it was not considered a drug-induced skin finding.

Therefore, the coexistence of these two diseases should not be considered as coincidence as it may herald an underlying malignancy in its early stages.

Punch biopsy was performed after the clinical evaluation of the patient and low-potency topical steroid 


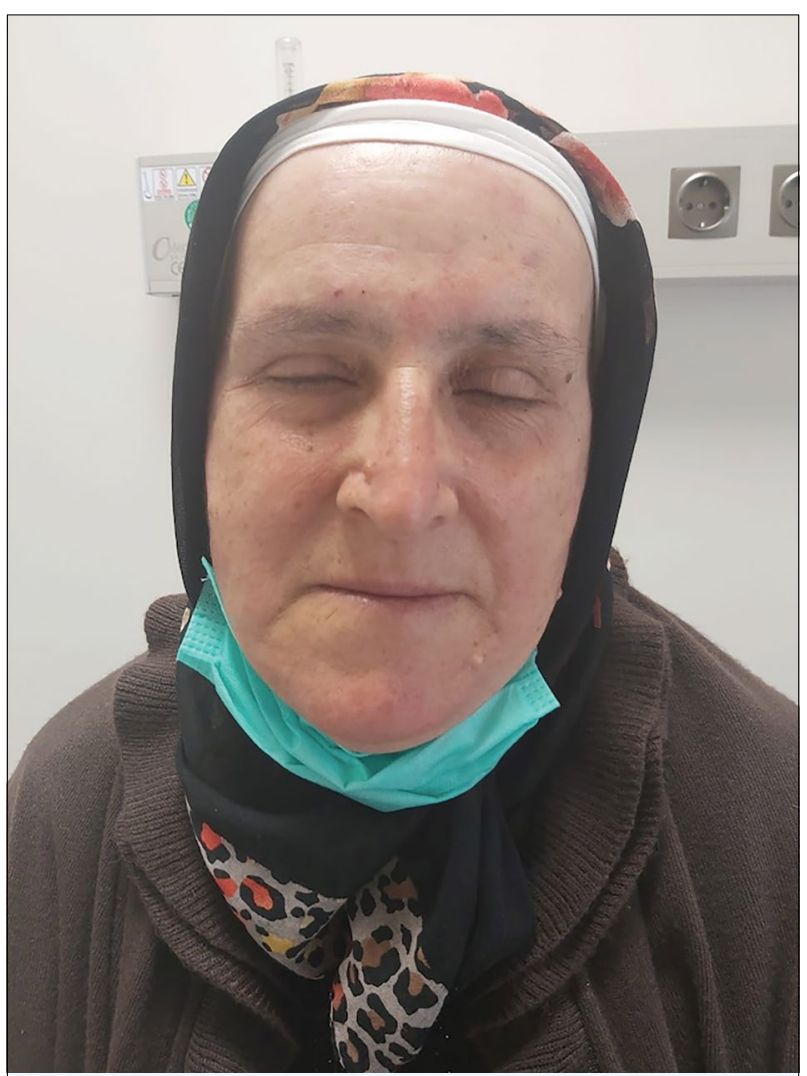

Fig. 4. Reduced erythema and infiltration on the face.

therapy was initiated for twice. During the patient's control examination in her third week, the SCLE lesion was partially regressed. Particularly, the erythema on her face was reduced and the plaques were thinning (Fig. 4). The patient's treatment continues in the oncology department.

\section{Discussion}

Presence of papulosquamous (33\%) and/or annular (97\%) lesions on the shoulders, forearms, neck, and upper torso; absent or mild systemic involvement; and the presence of anti-Ro (SSA) antibodies (70\%) are the most significant characteristics of SCLE.[1]

Paraneoplastic symptoms can be the first indication of an underlying malignancy. As many as $15 \%$ of patients diagnosed with a cancer have a concomitant paraneoplastic process at the time of diagnosis. [2]

An association between an internal malignancy and SCLE was reported in the literature since the 1980's. Lung, breast, head \& neck, gastric, liver, prostate, hepatocellular, laryngeal carcinoma, uterine, and esophageal adenocarcinoma and Hodgkin's lymphoma association with SCLE have been documented.[3-6] The tumor in the reported cases probably developed before approximately 3 to 36 months of the appearance of the eruption.[6] In our case, the cancer predated the dermatosis by one month. Nonscaly erythematous plaques first appeared on the face, then spread to the other areas involving the scalp, forearm, and dorsal of the hands. Her hair was intact, it fell off after the chemotherapy.

A small number of SCLE cases associated with breast carcinoma hs been revealed in the literature.[7] Gantzer et al.[8] reported two cases with breast cancer. One of these cases presented with an acute erythematous and squamous eruption after a relapsing cancer. The second patient was admitted for an erythematous, photo-distributed eruption occurring after a second breast cancer relapse and five months after the initiation of docetaxel. Regression of the skin lesions with a new chemotherapy treatment suggests that these cutaneous lesions may be associated with breast cancer.

For a dermatosis to be considered as paraneoplastic, it must occur simultaneously with malignancy, should have a parallel course with the malignancy, and regress with the treatment applied for cancer. The treatment of our patient continues in the oncology department. We thought that the improvement observed in the skin lesions might be due to the patient's chemotherapy and we planned to follow-up the patient for skin lesions for a while to confirm these lesions as paraneoplastic.

\section{Conclusion}

The recognition of paraneoplastic dermatosis is important because its determination can lead to the early diagnosis and treatment of the malignancy. It can also be used as a visible tumor marker.

Informed consent: Written informed consent was obtained from the patient for the publication of the case report and the accompanying images.

Peer-review: Externally peer-reviewed.

Conflict of Interest: No conflict of interest to declare.

Financial Disclosure: No funding was received.

Authorship contributions: Concept - S.B.D.; Design S.B.D.; Supervision - S.B.D.; Materials - S.B.D.; Data collection \&/or processing - S.B.D.; Analysis and/or interpretation - S.B.D, B.Y., A.K.; Literature search - S.B.D. Ö.A.S., P.K.; Writing - S.B.D., B.Y., A.K.; Critical review - S.B.D., B.Y., A.K.

\section{References}

1. Koritala T, Tworek, Schapiro B, Zolotarevsky E. Paraneoplastic cutaneous lupus secondary to esophageal 
squamous cell carcinoma. J Gastrointest Oncol 2015;6(3):61-5.

2. Bojinca V, Janta I. Rheumatic diseases and malignancies. Maedica (Bucur) 2012;7(4):364-71.

3. Loche F, Schwarze HP, Durieu C, Bazex J. A case of systemic lupus erythematosus associated with cancer of the lung: a paraneoplastic association? Br J Dermatol 2000;143(1):210-1.

4. McLean DI. Cutaneous paraneoplastic syndromes. Arch Dermatol 1986;122(7):765-7.

5. Chaudhry S, Murphy L, White I. Subacute cutaneous lupus erythematosus: a paraneoplastic dermatosis?
Clin Exp Dermatol 2005;30(6):655-8.

6. Jasim Z, Walsh M, Armstrong D. Subacute lupus erythematosus-like rash associated with oesophageal adenocarcinoma in situ. Clin Exp Dermatol 2007;32(4):443-5.

7. Shaheen B, Milne G, Shaffrali F. Subacute cutaneous lupus erythematosus associated with breast carcinoma. Clin Exp Dermatol. 2009;34(7):480-1.

8. Gantzer A, Regnier S, Cosnes A, Ortonne N, Wolkenstein $\mathrm{P}$, Bagot $\mathrm{M}$, et al. Subacute cutaneous lupus erythematosus and cancer: two cases and literature review. [Article in French]. Ann Dermatol Venereol 2011;138(5):409-17. 